\title{
Accuracy of four automatic QT measurement techniques in cardiac patients and healthy subjects
}

\author{
N B McLaughlin, R W F Campbell, A Murray
}

\begin{abstract}
Objective-To assess differences in the accuracy of automatic QT measurement in three subject groups, and to determine the influence of $T$ wave amplitude on these measurements.

Subjects-Standard simultaneous 12 lead electrocardiograms were acquired from 25 patients post myocardial infarction, 25 with arrhythmias, and 25 controls.

Design-Because there is not yet a standard automatic method for QT analysis, four different techniques were used. Manual QT measurements were used as the reference. QT was measured in two complexes by each technique in each lead, subject, and group.

Main outcome measure-The differences between reference and automatic QT measurements from the three subject groups were compared independently for the four techniques. The $T$ wave amplitudes for each of the groups were also compared.

Results-Variability of the automatic QT measurements, relative to the manual reference, in the cardiac patients was $2 \cdot 1$ times that in the controls $(P<0.005)$. Mean $T$ wave amplitude was lower (by a factor of two) for the cardiac patients compared with the controls $(P<0.01)$. No simple relation between $T$ wave amplitude and the difference between automatic and manual QT measurements was found, although the difference was $2 \cdot 2$ times greater for absolute $T$ wave amplitudes of less than $0.25 \mathrm{mV}(P<0.001)$.

Conclusions-Automatic QT measurement techniques are less accurate in cardiac patients than in controls. Measurements from T waves with amplitudes less than $0.25 \mathrm{mV}$ are less reliable.
\end{abstract}

(Heart 1996;76:422-426)

Keywords: QT measurement; repolarisation; automatic ECG measurement

An abnormally prolonged QT interval or abnormal QT dispersion are important noninvasive predictors of risk in cardiac patients.
Long QT syndrome is associated with QT dispersion and increased risk of cardiac sudden death through ventricular arrhythmias. ${ }^{12}$ An increased QT dispersion is associated with an adverse progress in heart failure ${ }^{3}$ and in hypertrophic cardiomyopathy. ${ }^{4}$ It is associated with a risk of ventricular fibrillation in acute myocardial infarction ${ }^{5}$ and has been linked with a risk of drug arrhythmogenesis. ${ }^{6}$ Manual measurement of the QT interval in all 12 leads of an electrocardiogram is tedious, and an automatic process would be welcome. Most modern electrocardiogram recording machines automatically measure the QT interval, and some measure it in all 12 leads. However, there is no standardised algorithm for QT measurement. This has led to a range of different automatic QT measurement algorithms being developed ${ }^{7}{ }^{8}$ or used by both industry and the research community. ${ }^{9-11}$ We showed important differences in QT measurement when different automatic techniques were used in subjects with normal electrocardiograms. ${ }^{12}$ The differences were dependent on the technique, and also on the parameters of each technique (threshold and isoelectric levels) and the electrocardiogram filtering used.

Electrocardiograms from cardiac patients often demonstrate different $\mathrm{T}$ wave amplitudes and morphologies relative to normal electrocardiograms. It is likely that the accuracy of automatic measurement techniques would be influenced by such features. The hypothesis of this study was that the accuracy of automated QT measurement is influenced by the presence of cardiac disease. Specifically our aims were to assess four automatic QT measurement techniques in two clinical subject groups and compare the results with those from a control group, and to assess the impact of the differences in $\mathrm{T}$ wave amplitude on automatic QT measurement.

\section{Methods}

\section{DATA COLIECTION}

12 lead electrocardiograms were obtained from cardiac patients referred for routine assessment. There were two disease categories:

Post myocardial infarction-Electrocardiograms obtained from patients at any time between their acute hospital admission and several years post infarction. 
Arrhythmia-Electrocardiograms recorded from patients with known ventricular or supraventricular arrhythmias but excluding atrial fibrillation.

Normal electrocardiograms were also obtained from healthy volunteers with no history of heart disease. ${ }^{12}$ Each group contained 25 subjects, and the group types were chosen to ensure that a wide range of $T$ wave morphologies was available for analysis.

Electrocardiogram acquisition and processing techniques were identical for the patients and the normal controls. Subjects lay in a semi-recumbent and relaxed state. The standard 12 lead electrocardiograms were amplified (gain 1000, bandwidth $0.05-100 \mathrm{~Hz}$ ) and sampled using a personal computer (Opus 386). A 12 bit analogue to digital conversion card (Metrabyte DAS16) provided a resolution of $2.44 \mu \mathrm{V}$. The 12 channels of electrocardiogram signal were acquired simultaneously at a sample rate of $500 \mathrm{~Hz}$. From each electrocardiogram, a section free from baseline artefact and containing two full

Figure 1 Main features of the automatic $Q T$ measurement techniques: TH (threshold level interception), DTH (differential electrocardiogram and threshold intersection), SI (slope intercept with isoelectric level), and PSI (peak slope intercept with isoelectric level). Full descriptions of the techniques are given in reference 12.
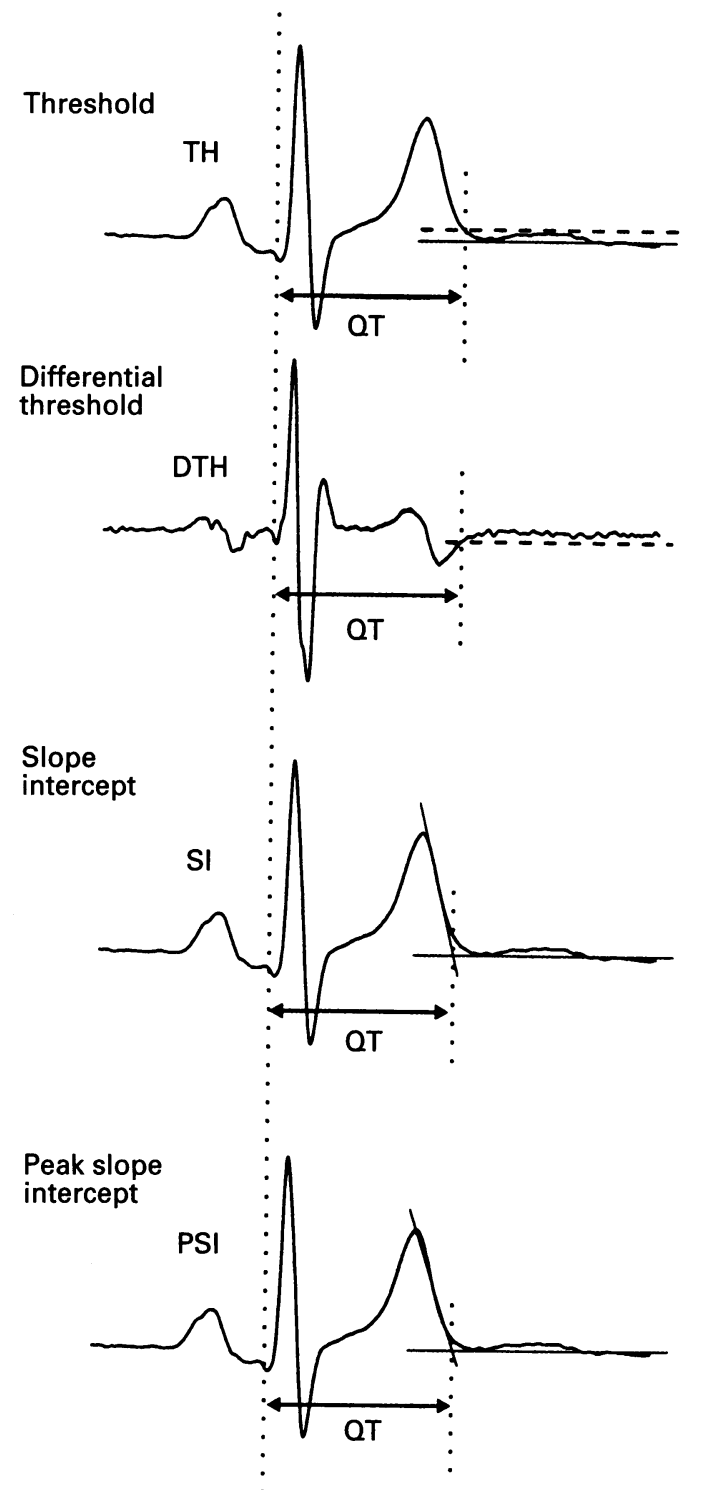

- Isoelectric level cardiac electrical cycles was selected, giving 600 PQRST complexes for each subject group (25 subjects $\times 12$ leads $\times$ two complexes) for manual and automatic analysis.

\section{MANUAL ANALYSIS}

Data plotting - The electrocardiograms were plotted to paper using a Hewlett Packard Laserjet III with a resolution of 118 dots per $\mathrm{cm}$. The plots had a vertical (voltage) scale equivalent to $10 \mathrm{~mm} \mathrm{mV}^{-1}$ and a horizontal (time) scale equivalent to $50 \mathrm{~mm} \mathrm{~s}^{-1}$.

QT measurement-Manual measurement was performed by an experienced researcher using a previously described standard and validated technique, ${ }^{13}$ in which the $R$ wave onset and $T$ wave offset on each electrodardiogram complex were identified using a digitising tablet.

\section{AUTOMATIC QT MEASUREMENT}

Electrocardiogram filtering - The bandwidth of the acquired electrocardiograms, determined by the analogue amplification system, was $0 \cdot 05-100 \mathrm{~Hz}$. After acquisition, using a low pass digital filter (2nd order Butterworth recursive filter) the electrocardiograms were further filtered to a bandwidth of $0.05-40 \mathrm{~Hz}$ to reduce myopotential and electrical noise.

Automatic feature identification-Software, described elsewhere, ${ }^{12}$ was used to identify the following: (a) the location in time of the $\mathrm{P}, \mathrm{R}$, and $T$ wave peaks and the $R$ wave onset, $(b)$ the isoelectric level of the TP segment, $(c)$ the amplitude of the $T$ waves relative to the isoelectric level, and (d) the magnitude and location of the maximum slope of the final component of the $T$ wave. $T$ wave peak was identified for all complexes, including those of very low amplitude. The $R R$ interval was calculated as the mean of the two $R R$ intervals enclosing the $T$ waves analysed. In addition, an upper limit for automatic QT measurement was set as the expected QT interval, calculated using Bazett's formula ${ }^{14}$ plus one fifth of the $\mathrm{RR}$ interval, and measurements beyond that limit were classed as failed.

$Q T$ measurement-For automatic determination of the $T$ wave end we used four techniques (fig 1), described elsewhere. ${ }^{12}$ The TH (threshold) and DTH (differential threshold) techniques determine the $T$ wave end as the intersection of a threshold level with the $T$ wave and the differential of the $T$ wave respectively. The threshold levels were calculated as a fraction $(0 \cdot 1)$ of the amplitude of the $T$ wave or differential $\mathrm{T}$ wave. Threshold crossing points were determined using a left to right scan of the data from the waveform peaks. For the $\mathrm{TH}$ technique the $\mathrm{T}$ wave amplitude and threshold level were calculated relative to the isoelectric level. The other two algorithms were based on slope features of the $T$ wave. Technique SI (slope intercept) identified the end of the $T$ wave as the intercept of the isoelectric level and a line tangential to the point of maximum $\mathrm{T}$ wave slope. The PSI technique (peak slope intercept) calculated the end of the $T$ wave as the intersection point between the isoelectric level and the line which passes 
Figure 2 Mean and standard deviation of automatic $Q T$ differences for the three subject groups.

Figure 3 Mean and absolute $T$ wave amplitudes for the three subject groups. standard deviation of
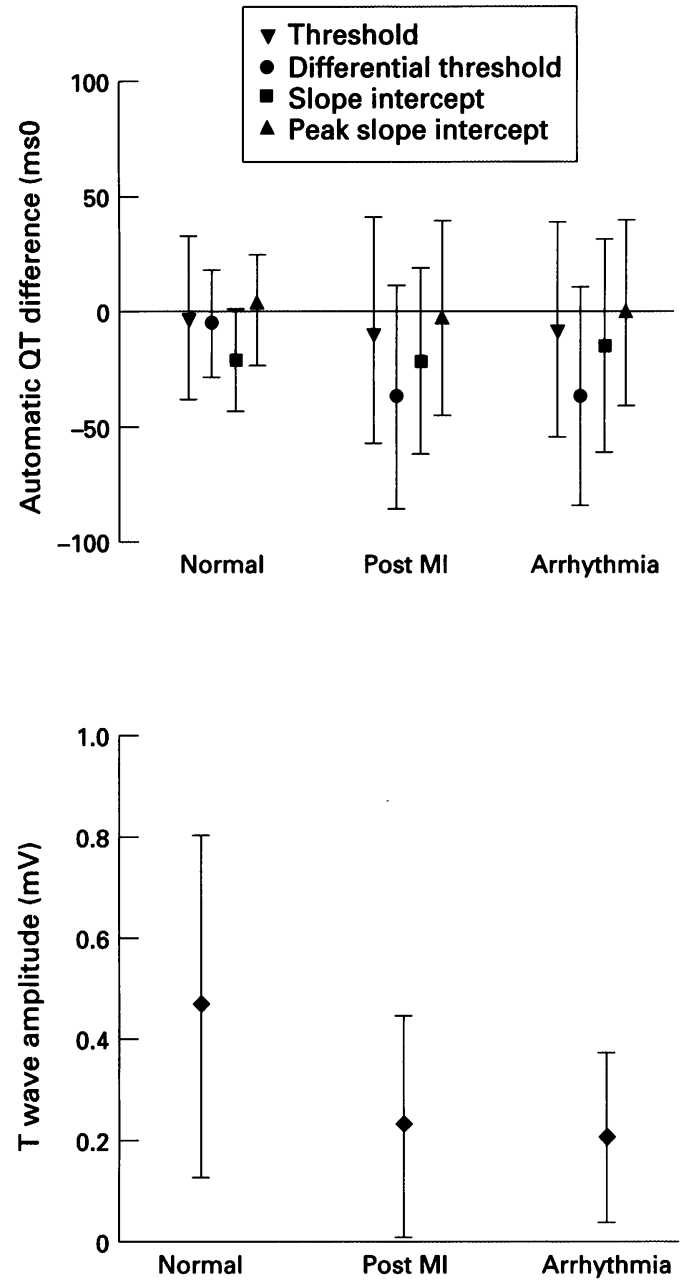

Normal

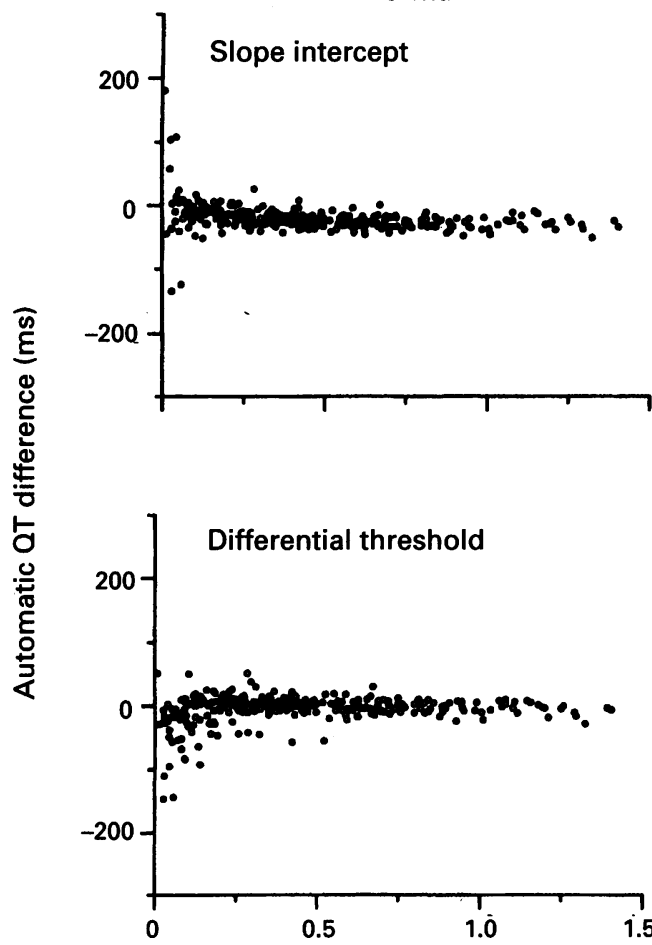

through the peak of the $T$ wave and the point of maximum $T$ wave slope.

DATA ANALYSIS

The mean of the two measured QT intervals was calculated for each lead. From the manual QT and four automatic QT measurements, the four automatic QT difference values (defined as the difference between automatic and manual measurement values for a given QT) were obtained. A negative automatic QT difference indicates an automatic measurement less than the corresponding manual measurement. For each automatic technique the mean and standard deviation of the automatic QT difference $\left(\operatorname{mean}_{\mathrm{AQD}}\right.$ and $\left.\mathrm{SD}_{\mathrm{AQD}}\right)$ across all $\mathrm{QT}$ values were calculated for each group. The mean and standard deviation of the absolute $T$ wave amplitudes for each group were also calculated.

Non-parametric tests (Mann-Whitney) were used to compare differences in automatic QT and $T$ wave amplitude between groups. The $F$ test was used to compare variances. Since measurements made across the electrocardiogram leads in a single subject are not completely independent, the degrees of freedom used in the statistics were based on the number of subjects in each group (25) rather than the number of QT values (300).

\section{Results}

The number of failed measurements within each subject group for each technique was low. In most cases zero fails occurred, and
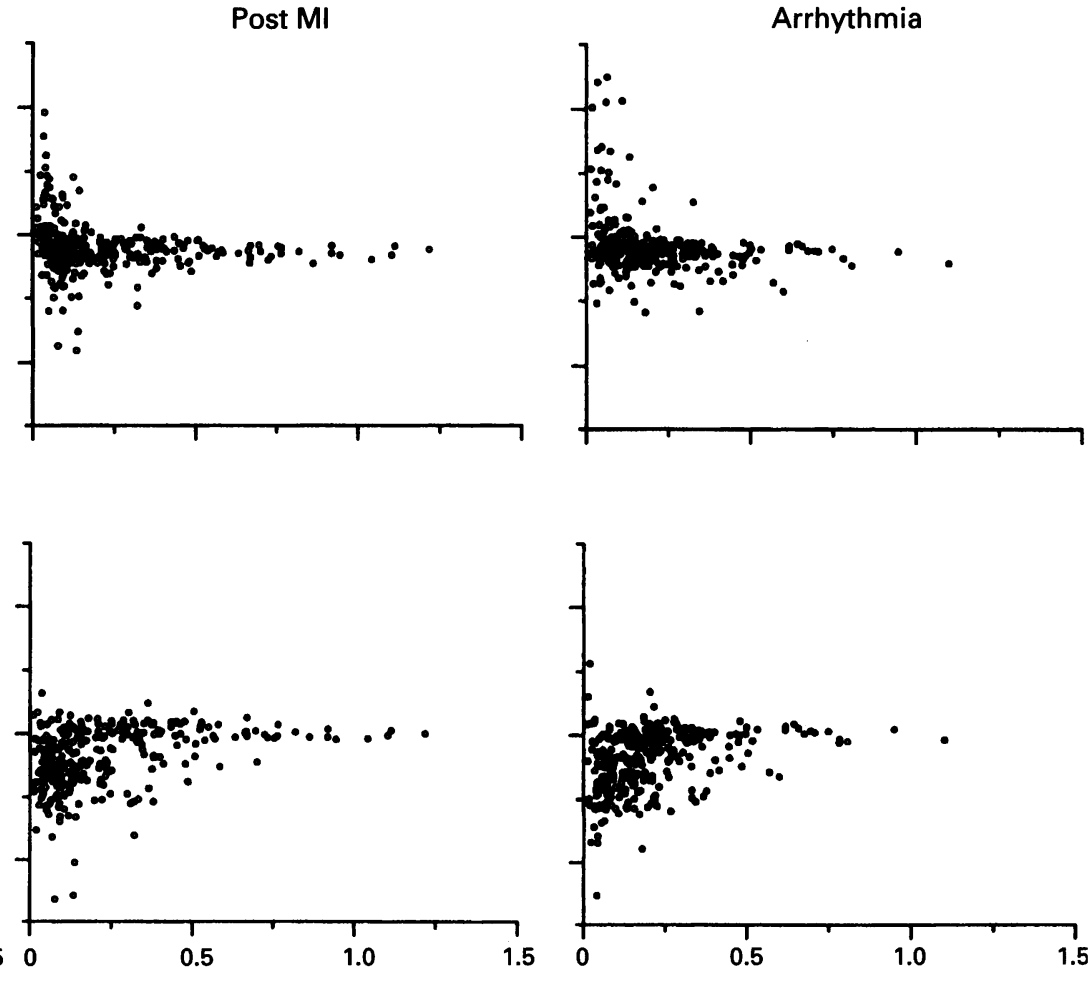

T wave amplitude $(\mathrm{mV})$

Figure 4 Scatter plots of automatic QT difference versus absolute $T$ wave amplitude for techniques SI (slope intercept) and DTH (differential threshold) for the three subject groups. (Note: three values where T wave amplitude was greater than $1.5 \mathrm{mV}$ have been omitted from the scatter plots of the normal group.) 
never exceeded four (out of a possible 300) for any given technique.

Figure 2 shows that there were greater differences between the normal subjects and cardiac patients than between the two cardiac patient groups. Mean $_{\mathrm{AQD}}$ was not significantly different between groups for most techniques. The exception was technique DTH where mean $_{\mathrm{AQD}}$ was lower $(\mathrm{P}<0.05)$ in both the post myocardial infarction and arrhythmia groups (by $-33 \mathrm{~ms}$ and $-32 \mathrm{~ms}$ respectively relative to the normal group). $\mathrm{SD}_{\mathrm{AQD}}$ was significantly greater for all techniques in the cardiac patients than in the normal subjects $(\mathrm{P}<$ 0.005 ) with the exception of technique $\mathrm{TH}$ in the arrhythmia group. The measurement variability in the cardiac patients was $2 \cdot 1$ times that in the normal subjects.

Figure 3 shows that mean absolute $T$ wave amplitude was significantly greater $(P<0.01)$ in the normal subjects than in the cardiac patients (times 2.0 with infarct group, times $2 \cdot 2$ with arrhythmia group). High automatic QT difference values tended to be associated with smaller $\mathrm{T}$ wave amplitudes. When $\mathrm{T}$ waves smaller than $0.25 \mathrm{mV}$ were compared with those greater than $0.25 \mathrm{mV}$ the overall $\mathrm{SD}_{\mathrm{AQD}}$ ratio was $2.2(\mathrm{P}<0.001)$. The ratio for each technique was DTH $1 \cdot 8$, TH $2 \cdot 0$, PSI $2 \cdot 2$, and SI $2 \cdot 7$. Figure 4 illustrates this with scatter plots of automatic QT difference for two automatic techniques (SI and DTH, the highest and lowest ratios respectively) against $\mathrm{T}$ wave amplitude for the three subject groups.

\section{Discussion}

Automatic QT measurements were more variable (relative to the manual reference) in cardiac patients than in normal subjects. This confirms our hypothesis that the performance of an automatic technique is influenced by the subject group under analysis. This has important implications for automatic QT measurement techniques which have been validated using only normal electrocardiograms.

There are clear differences between the normal subjects and cardiac patient groups in both the mean $T$ wave amplitude and the $\mathrm{SD}_{\mathrm{AOD}}$. This suggests a relation between amplitude and automatic QT difference. Inspection of the scatter plots (fig 4) reveals that even though this relation is not simple, the variability of results for all techniques and subject groups was greater for lower amplitude $T$ waves, and especially so for those less than $0.25 \mathrm{mV}$. The lack of a simple relation suggests that amplitude is only one of several factors influencing the accuracy of automatic QT measurement.

Because manual measurement was used as the reference, consideration must be given to the effect of the electrocardiogram on such measurement. We know from previous work that manual measurers are influenced by the amplitude of the $T$ wave in the normal electrocardiogram, ${ }^{13}$ with a small mean reduction of $8 \mathrm{~ms}$ in measured QT corresponding to a halving of the $T$ wave amplitude. This was associated with an insignificant change in measurement variability with half the $\mathrm{T}$ amplitude $(3 \%)$ or with superimposed noise $(14 \%)$. The small differences justify the use of manual measurement. Using an automatic technique as a reference would have removed it from the comparative assessment and the interpretation of results from automatic techniques with some features similar to the reference would have been difficult. It must, however, be accepted that lower amplitude and different morphology $T$ waves are likely to influence manual measurement. Automatic techniques, because they rely on specific $\mathrm{T}$ wave features, are less likely in practice to be able to cope with morphological and noise factors, particularly at low $T$ wave amplitudes. Our results demonstrate significantly increased variability in the clinical groups where $T$ waves were small. $T$ wave amplitudes less than $0.25 \mathrm{mV}$ do seem to present particular problems for automatic techniques and the results of measurements from such $T$ waves should therefore be treated with caution.

Of the four automatic measurement techniques studied, none provided any major advantage. If the techniques are to be used to determine group means, acceptable results can be obtained. It was interesting to note that the results from the PSI technique came closest to the mean manual measurements, perhaps because the technique pushed the measured end of the $T$ further out into the baseline than did the other techniques. It must, however, be remembered that different manual measurers can produce systematic differences of over $20 \mathrm{~ms}^{13}$ The technique which relied on differentiation (technique DTH) tended to underestimate QT intervals from cardiac patients compared with those from healthy subjects. This is likely to be because the differentiation process accentuates noise or because of peculiarities in the morphology of the end of the $T$ wave. Unfortunately, all techniques showed high measurement variability compared with manual methods, especially with small $T$ waves.

In conclusion, automated QT measurement techniques validated using electrocardiograms from healthy subjects should not be used to analyse electrocardiograms from cardiac patients without further validation. The variability of QT measurements in cardiac patients was twice that of normal subjects. Of the techniques examined, the one relying on differentiation introduced differences which could have an effect across leads and thus on dispersion, and hence cannot be recommended. In addition, the effect of small $\mathrm{T}$ waves has been quantified: there was between 1.8 and 2.7 times more variability for $\mathrm{T}$ waves less than $0.25 \mathrm{mV}$ than for those that were greater in amplitude. Current automatic measurements from small $\mathrm{T}$ waves should be treated with caution. These findings do not exclude the development of reliable automatic systems, but rather they underscore the need to develop new algorithms and techniques.

N B McLaughlin was supported by a British Heart Foundation grant. Mrs C Moore assisted with the collection and analysis of electrocardiograms used in this study. 
1 Somberg J, Tepper D, Wynn J. Prolonged repolarization: a historical perspective. Am Heart f 1985;109:395-8.

2 Day CP, McComb JM, Campbell RWF. QT dispersion: an indication of arrhythmia risk in patients with long QT indication of arrhythmia risk in patien

3 Barr CS, Naas A, Freeman M, Lang CC, Struthers AD. QT dispersion and sudden unexpected death in chronic heart failure. Lancet 1994;343:327-9.

4 Buja G, Miorelli M, Turrini P, Melacini P, Nava A. Comparison of QT dispersion in hypertrophic cardiomyopathy between patients with and without ventricular arrhythmias and sudden death. Am f Cardiol 1993;72: 973-6.

5 Higham J, Furniss SS, Campbell RWF. QT dispersion and components of the QT interval in ischaemia and infarction. Br Heart $₹$ 1995;73:32-6.

6 Hii JTY, Wyse DG, Gillis AM, Duff HJ, Solylo MA, Mitchell LB. Precordial QT interval dispersion as a marker of torsade de pointes. Disparate effects of Class 1a antiarrhythmic drugs and amiodarone. Circulation 1992;86:1376-82.

7 Laguna P, Thakor NV, Caminal P, Jane R, Yoon H-R, Bayes de Luna A, et al. New algorithm for QT interval analysis in 24-hour Holter ECG: performance and appli- cations. Med Biol Eng Comput 1990;28:67-73.

8 Algra A, le Brun $\mathrm{H}$, Zeelenberg $\mathrm{C}$. An algorithm for computer measurement of QT intervals in the 24 hour ECG. In: Computers in cardiology 1986. Los Alamitos: IEEE Computer Society Press, 1987:117-9.

9 Merri M, Benhorin J, Alberti M, Locati E, Moss AJ. Electrocardiographic quantitation of ventricular repolar zation. Circulation 1989;80:1301-8.

10 Mirvis DM. Spatial variation of QT intervals in norma persons and patients with acute myocardial infarction. $\mathscr{f}$ Amer Coll Cardiol 1985;5:625-31.

11 O'Donnell J, Lovelace E, Knoebel SB, McHenry PL. Behavior of the terminal T-wave during exercise in normal subjects, patients with symptomatic coronary artery disease and apparently healthy subjects with abnormal ST segment depression. $千$ Am Coll Cardiol 1985;5:78-84.

12 McLaughlin NB, Campbell RWF, Murray A. Comparison of automatic QT measurement techniques in the normal of automatic QT measurement techniques in the normal

13 Murray A, McLaughlin NB, Bourke JP, Doig JC, Furniss SS, Campbell RWF. Errors in manual measurement of QT intervals. Br Heart f 1994;71:386-90.

14 Bazett HC. An analysis of the time-relations of electrocardiograms. Heart 1920;7:353-70. 